\title{
DNA methylation alterations of AXIN2 in serrated adenomas and colon carcinomas with microsatellite instability
}

\author{
Yuta Muto ${ }^{1 \dagger}$, Takafumi Maeda ${ }^{1 \dagger}$, Koichi Suzuki $^{1 *}$, Takaharu Kato ${ }^{1}$, Fumiaki Watanabe ${ }^{1}$, Hidenori Kamiyama ${ }^{1}$, \\ Masaaki Saito ${ }^{1}$, Kei Koizumi ${ }^{2}$, Yuichiro Miyaki ${ }^{2}$, Fumio Konishi ${ }^{3}$, Sergio Alonso ${ }^{5}$, Manuel Perucho ${ }^{4,5,6^{*}}$ \\ and Toshiki Rikiyama ${ }^{1}$
}

\begin{abstract}
Background: Recent work led to recognize sessile serrated adenomas (SSA) as precursor to many of the sporadic colorectal cancers with microsatellite instability (MSI). However, comprehensive analyses of DNA methylation in SSA and MSI cancer have not been conducted.

Methods: With an array-based methylation sensitive amplified fragment length polymorphism (MS-AFLP) method we analyzed 8 tubular (TA) and 19 serrated (SSA) adenomas, and 14 carcinomas with (MSI) and 12 without (MSS) microsatellite instability. MS-AFLP array can survey relative differences in methylation between normal and tumor tissues of 9,654 DNA fragments containing all Not/ sequences in the human genome.

Results: Unsupervised clustering analysis of the genome-wide hypermethylation alterations revealed no major differences between or within these groups of benign and malignant tumors regardless of their location in intergenic, intragenic, promoter, or 3' end regions. Hypomethylation was less frequent in SSAs compared with MSI or MSS carcinomas. Analysis of variance of DNA methylation between these four subgroups identified 56 probes differentially altered. The hierarchical tree of this subset of probes revealed two distinct clusters: Group 1, mostly composed by TAs and MSS cancers with KRAS mutations; and Group 2 with BRAF mutations, which consisted of cancers with MSI and MLH1 methylation (Group 2A), and SSAs without MLH1 methylation (Group 2B). AXIN2, which cooperates with APC and $\beta$-catenin in Wnt signaling, had more methylation alterations in Group 2, and its expression levels negatively correlated with methylation determined by bisulfite sequencing. Within group 2B, low and high AXIN2 expression levels correlated significantly with differences in size $(P=0.01)$ location $(P=0.05)$ and crypt architecture $(P=0.01)$.

Conclusions: Somatic methylation alterations of AXIN2, associated with changes in its expression, stratify SSAs according to some clinico-pathological differences. We conclude that hypermethylation of $M L H 1$, when occurs in an adenoma cell with BRAF oncogenic mutational activation, drives the pathway for MSI cancer by providing the cells with a mutator phenotype. AXIN2 inactivation may contribute to this tumorigenic pathway either by mutator phenotype driven frameshift mutations or by epigenetic deregulation contemporary with the unfolding of the mutator phenotype.
\end{abstract}

Keywords: Colon sessile serrated adenoma, Microsatellite instability, BRAF mutation, DNA methylation, AXIN2

\footnotetext{
* Correspondence: ksuzbnhm@yahoo.co.jp; mperucho@imppc.org

${ }^{\dagger}$ Equal contributors

'Department of Surgery, Saitama Medical Center, Jichi Medical University,

1-847, Amanuma-cho, Omiya-ku, Saitama 330-8503, Japan

${ }^{4}$ Sanford-Burnham Medical Research Institute (SBMRI), 10901 North Torrey

Pines Road, La Jolla, California, USA

Full list of author information is available at the end of the article
} 


\section{Background}

Recent advances in colon cancer research have revealed a new pathological pathway distinct from the traditional pathway, the tubular adenoma-carcinoma sequence [1]. This alternative pathway has been recognized as the serrated pathway, in which sessile serrated adenoma (SSA) replaced the traditional tubular adenoma as the precursor lesion of a subset of colorectal cancer [2].

SSA was identified as a new entity by Torlakovic et al. in 1996 [3] and later classified in a new category, the serrated polyps [2]. The serrated polyps include hyperplastic polyps, traditional serrated adenomas and sessile serrated adenomas, the characteristics of which are serrated structure in the crypt epithelium [4-6]. Serrated polyp nomenclature is evolving and interpretation of the literature is complicated by differing interpretations of the morphological features of serrated polyps. Even among expert gastrointestinal pathologists there is significant interobserver variability in classification $[7,8]$.

Regardless of the difficulty in the definition, recent research efforts led to recognize that serrated polyps, especially SSA seemed to be precursor to many of the sporadic colorectal cancers with microsatellite instability (MSI) [9]. Mismatch repair deficiency leads to the accumulation of hundred of thousands of somatic mutations in microsatellite sequences [10]. This mutator phenotype defined a specific molecular pathway for colon cancer because the mutated cancer genes are in general different than those from cancers without MSI $[10,11]$. SSAs and MSI cancers were reported to exhibit similar features including predominant location in the proximal colon, high BRAF and low KRAS mutation and enhanced DNA hypermethylation [12-17].

Somatic hypermethylation of $\mathrm{CpG}$ islands in some genes includes the silencing of the MLH1 mutator gene and thus underlies many of the MSI sporadic cancers. Some investigators conferred distinctive phenotypic and biological properties to the tumors displaying a so-called CpG island methylator phenotype (CIMP), which was viewed as preceding the development of a subset of MSI colon cancers $[18,19]$. However, no apparent bimodal distribution was seen for the somatic hypermethylation alterations in gastrointestinal cancers $[20,21]$ thus challenging the CIMP hypothesis. Nearly 15 years later, the CIMP concept, despite the publication of many CIMP papers (reviewed in [22]) still awaits for a clear definition, including a stable set of CIMP markers, as well as for identification of the underlying methylator gene(s) [22,23].

Despite of the elusive CIMP entity, the importance of somatic hypermethylation as responsible for the silencing of several tumor suppressors and the $M L H 1$ mutator gene, and as a consequence the resulting MSI mutator phenotype, is highlighted by the evidence that SSA display DNA methylation alterations that are frequently observed in MSI cancer [9,24-26]. However, comprehensive analyses of methylation alterations in SSA and MSI cancer have not been conducted.

Methylation sensitive amplified fragment length polymorphism (MS-AFLP) is a fingerprinting technique developed by Yamamoto et al. as a tool to analyze DNA methylation in hundreds of loci simultaneously [27,28]. The approach utilized NotI restriction endonuclease for targeting methylation changes in any of the two CpG sites within its recognition sequence GCpGGCCpGC. Because nearly half of all NotI sites (44\%) are located in or adjacent to CpG islands, while the rest are located outside, MS-AFLP enabled to detect both relative DNA hypermethylation and hypomethylation somatic alterations throughout the genome. Comparing the intensity of the fingerprint bands from normal and tumor tissue DNA provided an unbiased insight of the complex picture of those epigenetic alterations. Employing this technique for the study of colorectal cancer we demonstrated that the MSI phenotype was dominant over hypermethylation [21] and that some of the tumors without MSI could be rationalized by an ageassociated accumulation of DNA hypomethylation [23].

More recently, we developed a novel MS-AFLP arraybased platform containing probes consisting of 60-meroligonucleotides, which cover the sequences adjacent to all the 9645 NotI sites identified in the human genome [29]. In this study, we performed a comprehensive analysis of methylation alterations to characterize the epigenetic profiles of colon adenomas and carcinomas of different genotype and phenotype to identify genes shared by these different neoplasms.

\section{Methods}

\section{Patients and tissues}

Nineteen patients with sessile serrated adenoma (SSA), 8 with tubular adenoma (TA) and 26 with proximal colon cancer including 12 and 14 tumors with and without MSI, respectively were recruited in this study. These were from the series analyzed in our previous study, which had enough amount and high quality of DNA and RNA available for microarray analysis [17]. SSAs, TAs and colorectal cancer tissues were prospectively collected in Jichi Medical University Hospital and Jichi Medical University Saitama Medical Center. SSA and TA were obtained endoscopically and classified with two categories by the location, i.e., proximal and distal.

SSA was diagnosed by five architectural features; basal crypt serration, basal dilatation of the crypts, crypts that run horizontal to the basement membrane, crypt branching and surface villosity or papillarity as previously described [17,30-32]. When the endoscopically resected polyp exhibited two or more features was diagnosed as SSA. Lesions showing typical histological features of so-called "traditional serrated adenoma" [5] were excluded from the analysis. 
Colon cancer tissues were obtained from patients who underwent surgical treatment. In all of the lesions, a part of the tissue was taken in fresh and was frozen immediately for genetic analysis and the rest of the tissue was used for histological analysis. Proximal lesions were defined as proximal to splenic flexure, whereas distal lesions were defined as distal to splenic flexure. All colorectal cancer tissues were collected from proximal colon. Written informed consent for participation in the study was obtained from all participants. This study was approved by Jichi Medical University Institutional Review Board.

\section{DNA and RNA extraction}

DNA was extracted by DNeasy ${ }^{\circ}$ blood and tissue kit (Qiagen, Hilden, germany). Total RNA was extracted from tissue culture cell lines by TRIzol $^{\circledR}$ Plus RNA purification kit (Invitrogen, Carlsbad, CA, USA).

\section{$B R A F$ and KRAS mutation analysis}

$B R A F$ (T1799A) and KRAS mutations were determined by direct sequencing after polymerase chain reaction (PCR) amplification of exon 15 of the BRAF gene and codon 12 and 13 of the KRAS gene. For detection of the $B R A F$ mutation, genomic DNA obtained from fresh frozen samples was amplified using: forward, 5 ' -TCAT AATGCTTGCTCTGATAGGA-3' and reverse, 5' -GG CCAAAAATTTAATCAGTGGA-3' primers. For the detection of the KRAS mutation, the following primers were used: forward, 5'-CTGAAAATGACTGAATA TAAACTTGT-3' and reverse, 5'-ATATGCATATTA AAACAAGATTTACC-3' as described $[17,33,34]$. PCR products were purified on a YM-30 Microcon column (Millipore) and sequenced using the BigDye terminator v3.1 cycle sequencing kit on ABI Prism 3100 (both from Applied Biosystems, Tokyo).

\section{MSI analysis}

Genomic DNA was extracted from fresh frozen samples using the EZ1 DNA tissue kit (Qiagen, Tokyo, Japan) and was amplified by PCR using the monomorphic markers BAT25 and BAT26 as previously described [27]. PCR products were analyzed by Gene Scan using ABI Prism 3100, and the sample was scored showing MSI if there were additional peaks in the PCR products, or otherwise scored as microsatellite stable (MSS).

\section{MLH1 and CpG island methylation}

Combined bisulfite restriction analysis was performed to assess gene methylation using primers that were designed to amplify the regions around the transcription start sites of the target genes [35]. Bisulfite modification was performed using the Epitect Bisulfite kit (Qiagen), as described previously [36]. Genomic DNA $(1 \mu \mathrm{g})$ was used for conversion with the bisulfite reagent. The primer sequences, annealing temperatures and restriction enzymes utilized were identical to those previously described [37]. After digestion, products were electrophoresed on $2 \%$ agarose gels and stained with ethidium bromide. Methylation density was confirmed using the image analysis program Image $\mathrm{J}$, and positive methylation was defined when the methylation- sensitive restriction enzyme digested $\geq 10 \%$ of the DNA [37].

\section{Preparation, labeling and hybridization of DNA samples for MS-AFLP arrays}

The genome-wide methylation profile was determined by a high-throughput array-based analysis of methylation alterations. For this purpose, we introduced an arraybased approach of the methylation sensitive amplified fragment length polymorphism (MS-AFLP) fingerprinting method, which can survey most of the 9654 DNA fragments containing all NotI sequences in the genome, as previously described [27,38]. Genomic DNA was isolated by QIAamp DNA Mini Kit (QIAGEN, Hilden, Germany). The initial steps of the MS-AFLP were performed as previously described [27,38]. Briefly, $1 \mu \mathrm{g}$ of genomic DNA was digested overnight with 5 units of methylation-sensitive NotI (Promega, Madison, WI, USA) and 2 units of methylation-insensitive Mse I (NE Biolabs, Beverly, MA, USA) at $37^{\circ} \mathrm{C}$. Two pairs of oligonucleotides were annealed overnight at $37^{\circ} \mathrm{C}$ to generate NotI (5' -CTCGTAGACTGCGTAGG-3' and 5'-GGCCCCT ACGCAGTCTAC-3') and Mse I (5'-GACGATGAG TCCTGAG-3' and 5' -TACTCAGGACTCAT-3') specific adaptors.

The digested DNA was ligated in $1.25 \mu \mathrm{l}$ each of 5 $\mathrm{pmol} / \mu \mathrm{l}$ NotI and $50 \mathrm{pmol} / \mu \mathrm{l}$ Mse I adaptor using 1 unit of T4 DNA ligase (Promega) overnight at $16^{\circ} \mathrm{C}$. The adaptor-ligated template DNA was amplified by PCR using NotI (5'-GACTGCGTAGGGGCCGCG-3') and Mse I (5'-GATGAGTCCTGAGTAA-3') primers. The PCR mixture consisted of $6 \mathrm{ng}$ of NotI primer, $30 \mathrm{ng}$ of Mse I primer, $0.25 \mathrm{mM} \mathrm{dNTP}$, and 1.5 unit of AmpliTaq DNA polymerase (Applied Biosystems, Foster City, California, USA) in a final volume of $20 \mu \mathrm{l}$. The PCR started at $72^{\circ} \mathrm{C}$ for $30 \mathrm{~s}$ and $94^{\circ} \mathrm{C}$ for $30 \mathrm{~s}$, followed by 35 cycles of $94^{\circ} \mathrm{C}$ for $30 \mathrm{~s}, 52^{\circ} \mathrm{C}$ for $30 \mathrm{~s}$, and $72^{\circ} \mathrm{C}$ for $2 \mathrm{~min}$. The final extension was performed for $10 \mathrm{~min}$ at $72^{\circ} \mathrm{C}$. The reactions were then kept at $10^{\circ} \mathrm{C}$ until the amplified DNA fragments were isolated using a QIA PCR Clean-up kit (Qiagen). DNA was eluted into $50 \mu \mathrm{l}$ of elution buffer.

Prior to hybridization on the MS-AFLP arrays, the DNA samples were differentially labeled as previously described [27,38]. Briefly, fluorescently labeled fragments were prepared using the Bioprime labeling system (Invitrogen). Each sample of PCR-amplified DNA (50 ng/ 
$2.5 \mu \mathrm{l})$ was mixed with $5 \mu \mathrm{l}$ of water and $5 \mu \mathrm{l}$ of Random Primer Mix solution. The mixtures were boiled at $100^{\circ} \mathrm{C}$ for $2 \mathrm{~min}$, quickly placed on ice for $1 \mathrm{~min}$, and briefly centrifuged for $10 \mathrm{~s}$. Then $1 \mu \mathrm{l}$ of either CY5 Mix solution (1.56 mM each of dGTP, dATP and dTTP, $0.22 \mathrm{mM}$ dCTP, and $0.11 \mathrm{mM}$ Fluorolink CY5-dCTP) or CY3 Mix solution (1.56 mM each of dGTP, dATP, and dTTP, $0.22 \mathrm{mM}$ dCTP, and $0.11 \mathrm{mM}$ Fluorolink CY3-dCTP) was added. Fluorolink CY5-dCTP and CY3-dCTP were purchased from Amersham-Pharmacia. Klenow fragment of E. coli DNA polymerase was then added to a final concentration of $0.8 \mathrm{U}$ per $\mu \mathrm{l}$. The mixtures were incubated at $37^{\circ} \mathrm{C}$ for $1 \mathrm{~h}$ before adding $2 \mu \mathrm{l}$ of stop solution (0.5 M EDTA) to terminate the reaction.

The CY5 and CY3 fluorescently labeled DNA fragments were separated from the unincorporated dNTPs by filtration through Microcon YM-30 columns (Millipore, Bedford, MA, USA). Each sample was reconstituted with $1 \times \mathrm{TE}(\mathrm{pH} 8.0)$ to a final volume of $37 \mu \mathrm{l}$, and $2 \mu \mathrm{l}$ of each sample was taken to determine the yield of labeled genomic DNA and the specific activity after labeling and clean-up. Exposure of samples to light was minimized during all experimental procedures.

The Cy3 and Cy5 labeled DNA samples were mixed in a siliconized tube with $70 \mu \mathrm{l}$ of Agilent $2 \times \mathrm{Hi}-\mathrm{RPM}$ Buffer (Agilent, Santa Clara, CA, USA). The mix was heated at $95^{\circ} \mathrm{C}$ for $3 \mathrm{~min}$ and centrifuged at $6000 \times \mathrm{g}$ for $1 \mathrm{~min}$ to collect the sample at the bottom of the tube. One hundred and ten $\mu \mathrm{l}$ of hybridization sample mixture was applied slowly to the gasket slide into the Agilent SureHyb chamber base. Then, one microarray slide was placed onto the gasket slide, with the active side facing down. The SureHyb chamber was covered onto the slides, and the clamp assembly was slid onto both pieces. The assembled slide chamber was placed in a rotator rack inside a hybridization oven and rotated at $20 \mathrm{rpm}$ and hybridized at $65^{\circ} \mathrm{C}$ for 40 hours. After hybridization, array slides were washed with Oligo aCGH Wash Buffer 1 at room temperature for 5 minutes and Oligo aCGH Wash Buffer 2 at $37^{\circ} \mathrm{C}$ for $1 \mathrm{~min}$. To prevent Cy5 degradation by ozone, the slides were washed with acetonitrile for $30 \mathrm{sec}-$ onds and then with Stabilization and Drying Solution for 30 seconds. The arrays were scanned using an Agilent G2565BA DNA Microarray Scanner.

\section{Quantitative reverse transcription-PCR}

Tissue specimens were immediately added to RNA later (Ambion, Austin, TX, USA) and stored at $-80^{\circ} \mathrm{C}$ until DNA or RNA extraction. Total RNA was immediately treated with DNase I (Invitrogen, Carisbad, CA, USA) and reverse-transcribed using a Superscript II reverse transcriptase kit (Invitrogen) to prepare first-strand cDNA. The primer sequences for AXIN2 were 5 '-CTGGCTCCA GAAGATCACAAAG-3' (forward) and 5'-ATCTCCTC
AAACACCGCTCCA-3' (reverse). Thermal cycling conditions were $42^{\circ} \mathrm{C}$ for $60 \mathrm{~min}$ (cDNA synthesis), $95^{\circ} \mathrm{C}$ for $30 \mathrm{sec}$ (hot start), and then 40 cycles of $95^{\circ} \mathrm{C}$ for $5 \mathrm{sec}$, $60^{\circ} \mathrm{C}$ for $30 \mathrm{sec}$, and $72^{\circ} \mathrm{C}$ for $60 \mathrm{sec}$. The expression level of AXIN2 was determined using the fluorescence intensity measurements from the ABI 7900HT Real-Time PCR System Data Analysis Software. An ACTB fragment was amplified as an internal control.

\section{Bisulfite sequencing analysis}

DNA sequencing was performed after bisulfite modification, as previously described [36]. The primers for the bisulfite sequencing were 5 '-TTGTATATAGTTTAGYG GTTGGG-3' (forward) and 5'-AAATCTAAACTCCC TACACACTT -3' (reverse). PCR was performed for 45 cycles, consisting of denaturation at $95^{\circ} \mathrm{C}$ for $30 \mathrm{sec}$, annealing at $58^{\circ} \mathrm{C}$ for $30 \mathrm{sec}$, and extension at $72^{\circ} \mathrm{C}$ for $60 \mathrm{sec}$, followed by a final 7 -min extension at $72^{\circ} \mathrm{C}$ for all primer sets. The sequences were subjected to a BLAST search to determine their location in the genome.

\section{Statistics}

Fisher's exact was used to examine associations between two categorical variables. Continuous variable comparisons between two groups were performed with the Student's t-test for those variables following a normal distribution, or with the non-parametric Mann-Whitney-Wilcoxon test for those variables that do not follow a normal distribution. The level of statistical significance was set at $P<0.05$, unless otherwise specified. To determine the significant genes from multiple samples, variance of analysis (ANOVA) with Bonferroni correction was carried out using $\mathrm{MeV}$ [39], by which hierarchical clustering sample and gene trees were also drawn, simultaneously. The threshold of significance was determined by Bonferroni correction set at $P=0.05$. To account for the bias due to the partial gene representation in the MS-AFLP Array, all the gene enrichment analyses were performed using the list of the genes present in the array as a background, instead of the total number of genes in the human genome [38].

\section{Results}

\section{Clinicopathological and molecular features of samples}

The clinicopathological and molecular features of the four subgroups of tumors, tubular adenomas (TA) sessile serrated adenomas (SSA), and MSI and MSS carcinomas, are summarized in Table 1. Patient gender and stage were not significantly different between each group. Patients with MSI cancers were older than those with SSA $(\mathrm{P}=0.01)$. MSI associated with poorly differentiated phenotype. KRAS mutation was more prevalent in MSSs and TAs as compared to MSIs and SSAs $(\mathrm{P}<0.01)$, whereas BRAF mutation was preferentially observed in MSIs and SSAs as 
Table 1 Clinicopathological and molecular data of colon adenomas and carcinomas

\begin{tabular}{|c|c|c|c|c|c|}
\hline & TA $(n=8)$ & SSA $(n=19)$ & MSS $(n=12)$ & MSI $(n=13)$ & P-value ${ }^{3}$ \\
\hline Age & $65.4 \pm 4.4$ & $60.6 \pm 9.8$ & $62.2 \pm 8.2$ & $70.8 \pm 11.3$ & $0.019^{(a)}$ \\
\hline Gender (M/F) & $4 / 4$ & $13 / 6$ & $6 / 6$ & $6 / 7$ & 0.57 \\
\hline Duke's stage & NA & NA & $7 / 5$ & $11 / 2$ & 0.20 \\
\hline \multicolumn{6}{|l|}{$(A$ or $B / C)$} \\
\hline Grade $^{1}$ & NA & NA & $12 / 0$ & $9 / 4$ & 0.096 \\
\hline \multicolumn{6}{|l|}{ (W-M/P) } \\
\hline KRAS Mutations & $4 / 8$ & $0 / 19$ & $4 / 12$ & $0 / 13$ & 0.0011 \\
\hline \multicolumn{6}{|l|}{ (mut/total) } \\
\hline BRAF Mutations & $0 / 8$ & 19/19 & $4 / 12$ & $9 / 13$ & $2.2 \times 10^{-6}$ \\
\hline \multicolumn{6}{|l|}{ (mut/total) } \\
\hline hMLH1 Methylation (+/total) & $0 / 8$ & $0 / 19$ & $0 / 12$ & $5 / 13$ & $8.6 \times 10^{-4}$ \\
\hline Hypermethylation ${ }^{2}$ & $1.8 \pm 1.4 \%$ & $1.2 \pm 0.6 \%$ & $1.2 \pm 0.6 \%$ & $1.9 \pm 1.2 \%$ & $0.15^{(a)}$ \\
\hline Hypomethylation ${ }^{2}$ & $0.6 \pm 0.4 \%$ & $0.3 \pm 0.2 \%$ & $1.0 \pm 0.5 \%$ & $1.0 \pm 0.6 \%$ & $5.8 \times 10^{-4(a)}$ \\
\hline
\end{tabular}

${ }^{1}$ Tumor grade. Well (W), moderately (M) or poorly (P) differentiated.

${ }^{2}$ Hypermethylation and hypomethylation indicate the percentage of MS-AFLP array probes with values surpassing the hypermethylation and hypomethylation thresholds, respectively.

${ }^{3}$ For categorical data, $\mathrm{p}$-values were calculated by $\mathrm{x}^{2}$ test when comparing four groups, or by Fisher's exact test when comparing two groups. For continuous data, we applied one-way ANOVA followed by Tukey's HSD multi-hypothesis testing correction. The most Statistically significant $p$-value after correction corresponded always to the SSA vs MSI comparison ${ }^{(a)}$. In hypomethylation, a significant difference between SSA and MSS was also found (P=0.0014). P-values below 0.05 are in bold type.

NA: Not applicable.

compared to MSSs and TAs $(\mathrm{P}<0.01)$. MLH1 methylation was detected only in MSI carcinomas.

Genome-wide surveillance of methylation alterations by MS-AFLP revealed no significant differences between the 4 groups (Table 1 ). Also, there was no significant difference in the overall frequency of hypermethylation alterations (including intragenic and intergenic regions) and in the promoter and 3 'end regions (Figure 1). There was a borderline difference in methylation frequency at intragenic and 3 'end regions $(\mathrm{P}=0.035$ and $\mathrm{P}=0.041$, respectively) between SSAs and MSI carcinomas due to the higher number of alterations of the later (Additional file 1: Figure S1). Regarding hypomethylation, SSAs displayed fewer alterations than MSS and MSI carcinomas overall and in the different gene regions although the differences were more pronounced compared with the MSI carcinomas (Figure 1 and Additional file 1: Figure S1).

Since the MS-AFLP array covers all NotI sites in the genome, these results extend our previous findings with the MS-AFLP DNA fingerprinting lower resolution approach showing that the original method reflected a panoramic view of the somatic methylation alterations undergone by colon cancers at NotI sites.

\section{Differentially methylated loci in TA, SSA, MSS and MSI}

To identify the possible existence of distinct methylation profiles specific for each of the four different tumor subgroups, unsupervised hierarchical clustering was performed using 9,645 probe sets, but the results revealed no clear differences (data not shown). We, then, carried out an analysis of variance (ANOVA) to determine whether there were particular loci specifically associated with these tumor subgroups, especially with serrated adenomas. This analysis resulted in the identification of 56 distinctive probes, corresponding to 35 genes, 5 putative loci and 12 intergenic sequences (Figure 2 and Additional file 1: Table S1) that appeared altered differentially among these subgroups.

The ANOVA-constructed hierarchical tree revealed two distinct subsets of samples (Groups 1 and 2, Figure 2). Seven MSSs (58.3\%) and 2 MSIs (14.3\%) cancers, as well as 7 TAs $(87.5 \%)$ were assigned to Group 1, while 12 MSIs (85.7\%), all 19 SSAs (100\%), 5 MSSs (41.7\%) and 1 TA (12.5\%) to Group 2. Group 1 thus, consisted of many tumors participating in the tubular adenoma-carcinoma pathway (TAs and MSSs cancers), whereas Group 2 included many SSAs and MSI cancers (Figure 2).

\section{Distinct methylation profiles associates with SSAs including AXIN2}

The clustering into two distinct groups by the ANOVA approach allowed performing t-test analysis of these two groups (Additional file 1: Figure S2). The constructed hierarchical tree revealed distinct epigenetic profiles, one of which was shared by many of the MSI cancers and SSAs, and another, which was shared by MSS carcinomas and TAs. T-test also identified 168 probes that 

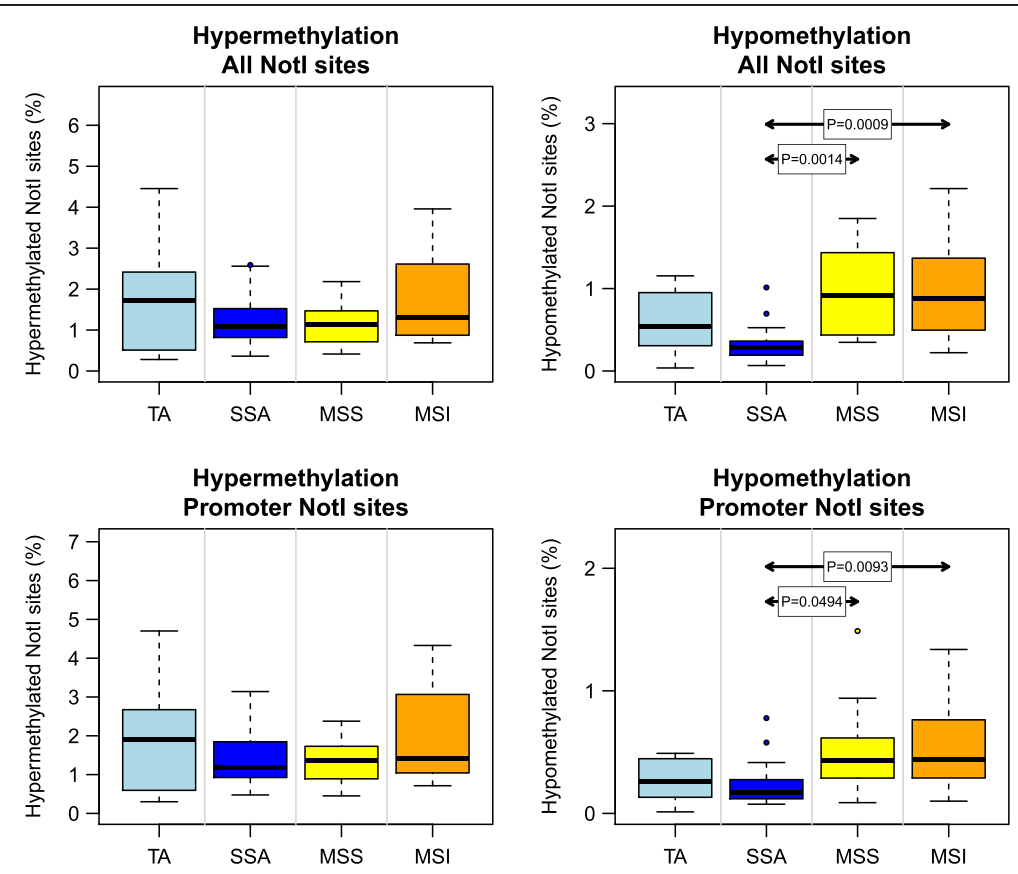

Figure 1 Frequency of hypermethylation (left) and hypomethylation (right) estimated by MS-AFLP arrays. TA, in light blue: tubular adenomas. SSA, in dark blue: sessile serrated adenomas. MSS, in yellow: microsatellite stable carcinomas. MSI, in orange: microsatellite instable carcinomas. Frequencies were calculated as the percentage of probes with log2 ratio value below -1.5 (for hypermethylation) or above 1.5 (for hypomethylation), after filtering the $30 \%$ lower-intensity probes from each array. Top graphs, results including all probes after filtering (13,515 probes per array). Bottom graphs, results considering only the probes within $\pm 2.5 \mathrm{~Kb}$ of the $5^{\prime}$ end of genes (range: 7,924 to 8,035 probes per array). P-values were calculated by one-way ANOVA followed by Tukey's HSD multi-hypothesis testing correction. Only p-values below 0.05 are shown.

distinguished these tumors of the serrated-MSI cancer pathway and the tubular adenoma-MSS carcinoma pathway (Additional file 1: Figure S2). AXIN2 was one of these genes, which displayed a distinct level of methylation alterations between the two groups (black bar at right margins of Figure 2 and Additional file 1: Figure S2).

Group 2 was further classified into two subgroups, one of which included most MSI cancers (71.4\%, Group$2 \mathrm{~A})$, and the other which contained all the SSAs (100\%, Group-2B) (Figure 2). Group 1 displayed high frequency of $K R A S$ mutation whereas Group 2 exhibited high frequency of $B R A F$ mutation (Figure 2 bottom). While Group 2 harbored many of MSI cancers and all SSAs, as expected, methylation of $M L H 1$ was only seen in MSI cancers in Group 2A. Group 2B was also subdivided into two groups, $2 \mathrm{~B}-1$ and $2 \mathrm{~B}-2$, according to the hierarchical tree (Figure 2 bottom).

\section{Expression of AXIN2 associates with methylation alterations}

ANOVA analysis identified AXIN2, which plays an important role in Wnt signaling pathway cooperating with $\mathrm{APC}$ and b-catenin, being more frequently altered in Group 2 that included the serrated adenomas. We measured the abundance of the corresponding AXIN2 mRNA in the original SSAs and MSI cancers (Group 2) by quantitative reverse transcription-polymerase chain reaction (RT-PCR). The expression levels were variable, with some tumors showing little or no expression, while others exhibited a relatively high expression (Figure 3 right).

When the expression levels of AXIN2 were compared between the different groups according to the hierarchical tree (Figure 2), the groups $2 \mathrm{~A}$ and $2 \mathrm{~B}-1$ exhibited low expression levels of $A X I N 2$, whereas group 2B-2 had significant higher levels of expression of AXIN2 (Figure 3, left). To examine whether the decreased levels of AXIN2 mRNA was linked to aberrant methylation, the degree of methylation alterations of $12 \mathrm{CpG}$ sites within the AXIN2 promoter region (Figure 3 middle) was assessed in 5 plasmid clones of each of several samples from groups 2A, 2B-1 and 2B-2. The AXIN2 promoter appeared more methylated in samples from Group 2A than in Groups 2B-1 and 2B-2 (Figure 3 middle). Also, a tumor cell line with high methylation (HCT116) exhibited lower expression of AXIN2 than another cell line (Caco2) with little methylation (Figure 3, top of middle and right panels).

\section{Expression of AXIN2 associates with different clinicopathological features of SSAs}

The clinicopathological features of SSAs were analyzed in regards to the observed differences in AXIN2 methylation 


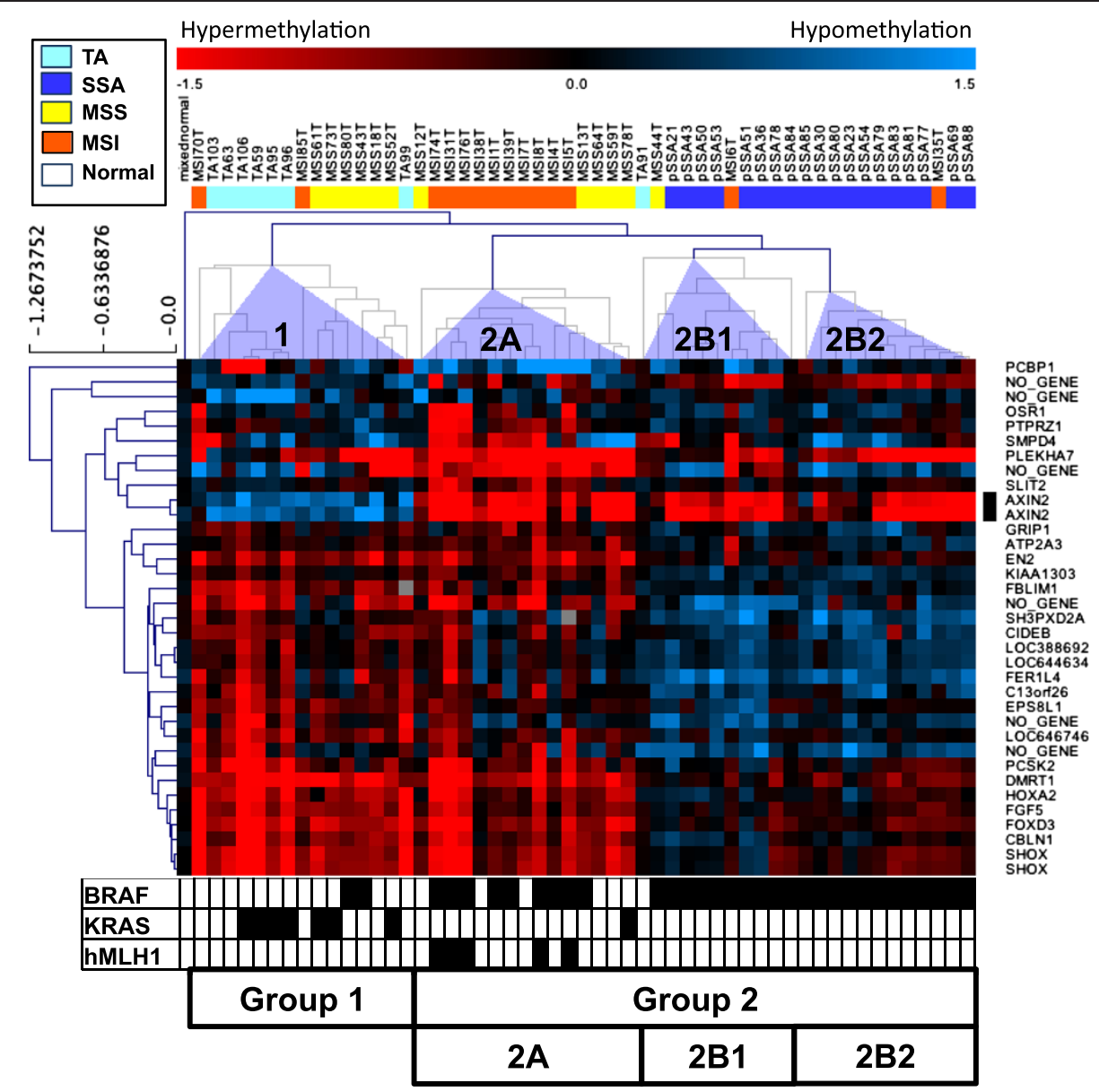

Figure 2 Clustering of the samples according to their methylation profile. Hypermethylation is indicated in red. Hypomethylation is indicated in blue. Samples are shown on top of the heatmap. In white, the normal tissue DNA mix used as reference. Colors for the four tumor groups are as in Figure 1. On the right side of the heatmap, the genes associated to MS-AFLP probes. AXIN2 probes are indicated in black. Clustering was performed by complete linkage using Pearson's correlation on a subset of MS-AFLP probes previously selected by ANOVA. Group 1 contains mostly tubular adenomas and MSS carcinomas. Group 2A contains the majority of MSI carcinomas, and 5 MSS carcinomas. All the sessile serrated adenomas are grouped in 2B1 and 2B2. Below the heatmap, in black, cases positive for mutation in BRAF or KRAS, or hypermethylation of $h M L H 1$.

and expression of the two subgroups $2 \mathrm{~B}-1$ and $2 \mathrm{~B}-2$. The results are shown in Table 2 . No differences were evident between these two groups in age, gender, presence of carcinoma in the adenoma, or mucinous phenotype. However, the adenomas with high methylation and low expression (Group 2B-1) were significantly smaller, were more distal, and exhibited less crypt branching than those adenomas with low levels of methylation and high levels of expression (Group 2B-2).

\section{Discussion}

In this study, genome-wide surveillance of hypermethylation alterations in NotI sites by MSFLP-array revealed that somatic hypomethylation was lower in SSAs compared with MSI or MSS carcinomas. These benign tumors also occurred in younger individuals compared with MSI carcinomas. This is consistent with the proposed hypothesis of demethylation as a gradual accumulation of methylation replication errors during aging [23] assuming SSAs being the precursors of the MSI carcinomas. In contrast, there were no major differences in global hypermethylation between these groups of benign and malignant tumors regardless of their location in intergenic, intragenic, promoter, or 3 'end regions. Unsupervised clustering analysis revealed no clear differences in the patterns of hypermethylatuon between or within the four different tumor groups. Only after applying an ANOVA approach was possible to discern that MSS cancers and TAs shared similar epigenetic features, so did MSI and SSA, as reported previously [12-17]. The study also disclosed distinct profiles of genes relevant for colorectal cancer such as homeobox genes, transcription factors, growth factors and genes in the Wnt signaling pathway, including AXIN2. 


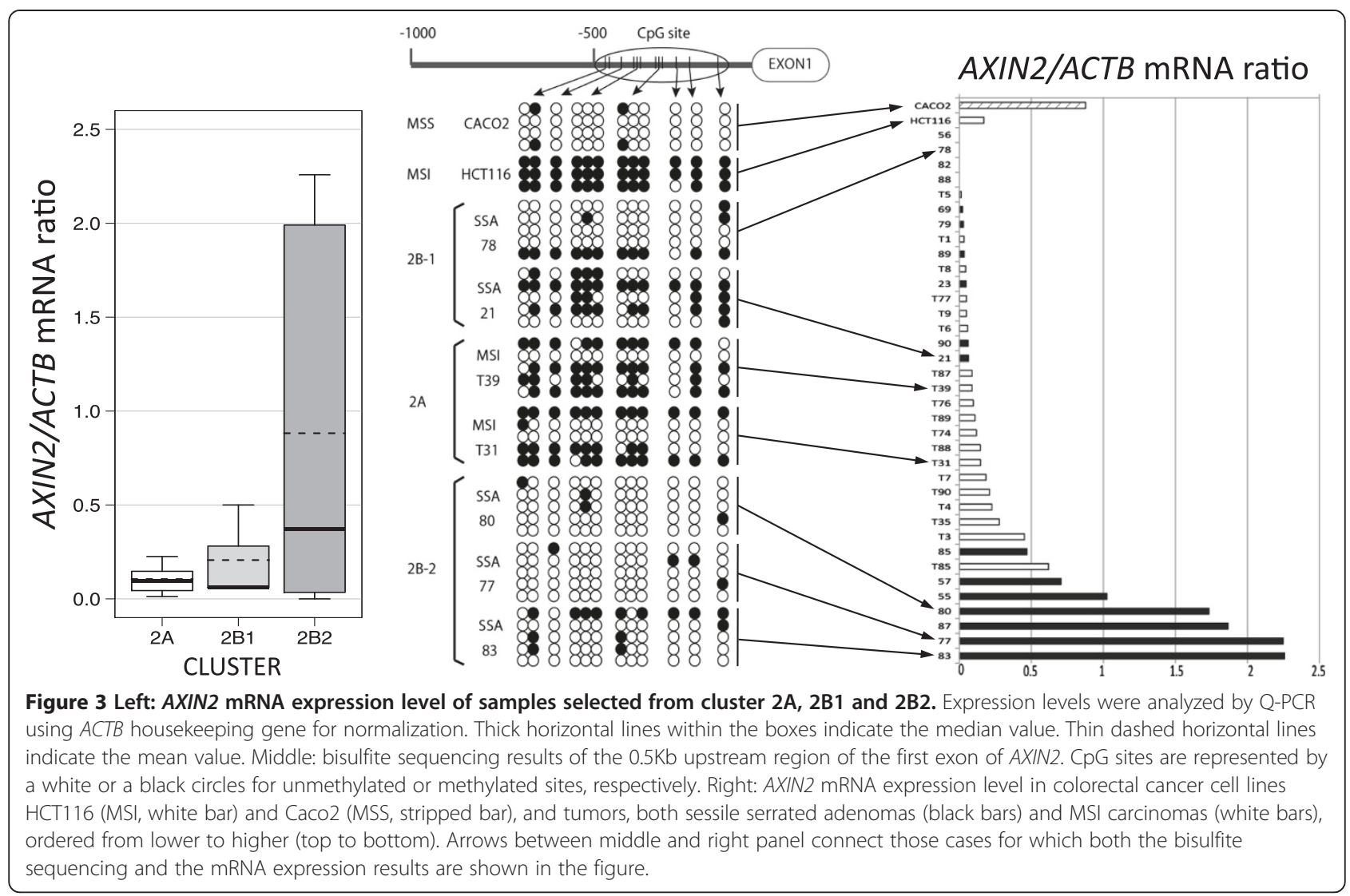

Several papers estimated the frequency of Wnt signaling activation in SSAs but they are controversial [40-44]. Possible explanations to account for the discrepancies may include that some SSAs were misdiagnosed and wrongly categorized due to the complication in the definition of serrated polyps [45]. Therefore, a standardized diagnosis of SSA formulated recently [30,32] was applied in this study.

Recent genome-scale exome sequencing analysis of 276 colorectal tumors, DNA copy number, promoter methylation and messenger RNA and microRNA expression conducted by the Cancer Genome Atlas project,

Table 2 Comparison of SSAs according to AXIN2 expression status

\begin{tabular}{|c|c|c|c|}
\hline & Group 2B-1 $(n=8)$ & Group 2B-2 $(n=11)$ & P-value ${ }^{4}$ \\
\hline Gender (Male/Female) & $4 / 4$ & $9 / 2$ & 0.32 \\
\hline Patient Age (years) & $62.8 \pm 9.9$ & $59.1 \pm 9.9$ & 0.44 \\
\hline (Range, Median) & $(46-74,63)$ & $(40-76,59)$ & \\
\hline Tumor Size (mm) & $9.4 \pm 1.9$ & $15.3 \pm 6.1$ & 0.01 \\
\hline (Range, Median) & $(6-12,10)$ & $(8-28,15)$ & \\
\hline Location $(\mathrm{C}-\mathrm{A} / \mathrm{T})^{1}$ & $3 / 5$ & $10 / 1$ & 0.041 \\
\hline $\begin{array}{l}\text { Crypt Branching }{ }^{2} \\
\text { (+/total) }\end{array}$ & $0 / 8$ & $8 / 11$ & 0.003 \\
\hline $\begin{array}{l}\text { Hypermucinous }{ }^{3} \\
\text { (+/total) }\end{array}$ & $8 / 8$ & $11 / 11$ & 1.00 \\
\hline $\begin{array}{l}\text { Carcinoma in adenoma } \\
(+/ \text { total })\end{array}$ & $0 / 8$ & $1 / 11$ & 1.00 \\
\hline
\end{tabular}

${ }^{1}$ Anatomical location. Cecum (C). Ascending (A). Transversal (T).

${ }^{2}$ Crypt branching was defined by the appearance of splits or fissures in the base of the crypts.

${ }^{3}$ Hypermucinous appearance.

${ }^{4} \mathrm{P}$-values were calculated by Fisher's exact test for categorical variables and unpaired t-test for continuous variables. $\mathrm{P}$-values below 0.05 are in bold type. 
[46] indicated that $92 \%$ of MSI cancer and $97 \%$ of MSS cancers exhibited at least one alteration of genes involved in the Wnt pathway including LRP5, FZD10, FAM123B, AXIN2, APC, CTNNB1 ( $\beta$-catenin), TCF7L2, $F B X W 7$ and SOX7. Thus, Wnt signaling pathway seems to play a critical role in colorectal carcinogenesis in general, although the spectrum of alterations may vary depending on the distinct oncogenic pathways.

AXIN was identified as a component of the complex in Wnt signaling pathway to regulate the levels of $\beta$ catenin along with the wild type of adenomatous polyposis coli $(A P C)$ gene [47]. AXIN1 plays as a scaffold protein on which the complex for phosphorylation of $\beta$ catenin by glycogen synthase kinase-3 $\beta$ (GSK-3 $\beta$ ) is assembled [48]. AXIN2 / Conductin was identified as an AXIN homolog, which also played a scaffold protein, and was found mutated in a subset of colorectal cancers $[47,49]$. AXIN1 appears to be a constitutive component of $\beta$-catenin degradation complex for maintenance of basal life activity while AXIN2 is considered to be an inducible component that is upregulated in response to increases in $\beta$-catenin levels and thus serves to limit the duration and intensity of the Wnt signal [50,51]. AXIN2 has been only found expressed in colon tissues (Additional file 1: Figure S3).

Epigenetic silencing of AXIN2 in MSI colon cancer was reported in 2006 [52]. However, aberrant methylation of AXIN2 in SSA has not been previously reported. In addition, we identified an apparent increase in methylation alterations of AXIN2 from SSAs to MSI carcinomas, suggesting that its expression deregulation by methylation associates with the serrated adenoma-MSI cancer pathway.

The hierarchical tree identified three clusters according to methylation profiles, MSI, SSAs epigenetically close to MSI and SSAs far from MSI. The expression levels of $A X I N 2$ in these three groups associated with the levels of methylation of AXIN2 in each group, respectively (Figure 3 ). Our results suggest that expanding of methylation in the promoter region of AXIN2 in SSAs lead to the suppression of the AXIN2 gene expression gradually, which contributes to a stepwise acquisition of the epigenetic features seen in MSI colon cancer. Koinuma et al. [52] reported that overexpression of AXIN2, either by treatment with $5^{\prime}$-azacytidine or by transfection with AXIN2 cDNA, resulted in rapid cell death in a MSI CRC cell line, which supports the functional significance of $A X I N 2$ changes in methylation and expression in our study. Dong et al. [24] reported progressive methylation of several genes during the serrated pathway. In contrast with the epigenetic silencing of AXIN2 in MSI colon cancer, up-regulation of AXIN2 mRNA was reported in MSS cancers. Indeed, in our study, AXIN2 was frequently hypomethylated in MSS cancers, suggesting that the epigenetic change of AXIN2 specifically associates with the MSI pathway for colon cancer. The fact that down-regulation is not always accompanied by methylation (Figure 3 ) shows that additional mechanisms may be at play to inactivate the suppressor function of the AXIN2 protein. For instance, frameshift mutations of AXIN2 in MSI colon cancers may be one such additional mechanism [49,53,54].

The epigenetic influence on MSI manifestation is shown by the hypermethylation and silencing of $M L H 1$ [55]. High level of hypermethylation has been also associated with MSI cancers $[19,56]$, and also in SSAs [9,13,15,16,24-26,57,58]. However, MLH1 methylation was not detectable in SSAs in contrast with the common presence observed in MSI cancers. This shows that the epigenetic silencing of $M L H 1$ is not involved in SSA development where it must occur sometime during the adenoma expansion. But silencing of $M L H 1$ then appears to drive the adenoma cells towards the carcinoma state by the generation of many subsequent mutations. The difference in age between the patients with SSAs and MSI carcinomas also supports this suggestion, implying a necessary additional step after SSA development for the accumulation of oncogenic mutations responsible for the carcinoma transition.

AXIN2 aberrant methylation appears to occur during adenoma growth like MLH1 methylation. The assumption here is that no methylation of $M L H 1$ is found at the SSA stage because once it occurs it may lead to the carcinoma transition in the absence of further clonal expansion, since mutator genes do not alter the growth properties of the cells. The association observed between aberrant methylation and down-regulation with small size SSAs without crypt branching could be interpreted assuming that the occurrence of $M L H 1$ methylation may speed the transition to carcinoma in the absence of a need for further expansion of the adenoma.

\section{Conclusions}

In conclusion, this study revealed that methylation aberrations likely play a role in the serrated adenoma-MSI carcinoma sequence in colon cancer. Although the samples in this study are too limited to draw definitive conclusions in some genetic or epigenetic comparisons, other differences were sufficiently large to reach statistical significance. $M L H 1$ silencing seem to occur in an already developed serrated adenoma by the previous occurrence of somatic mutation in the BRAF oncogene. Once the serrated adenoma has evolved, additional somatic alterations altering Wnt signaling, such as AXIN2 methylation or frameshift mutation, may contribute to the adenoma's further growth. Other genes besides AXIN2, were identified that exhibit methylation profiles shared between SSA and MSI CRC and would be 
interesting to further investigate how these genes work and interact with each other during the progression of colon cancer of the serrated adenoma-MSI carcinoma sequence. Nevertheless, when, contemporary with these somatic alterations, aberrant methylation of the $M L H 1$ gene occurs, this appear to be the determinant event in those cases that eventually progress to the carcinoma stage by providing the cells with a strong mutator phenotype.

\section{Additional file}

Additional file 1: Figure S1. Frequency of hypermethylation and hypomethylation of the different tumor groups, estimated by MS-AFLP arrays. Figure S2. Clustering of the samples according to their methylation profile. Figure S3. Gene expression pattern of the AXIN2 gene in human normal and cancerous tissues. Table S1. Loci with differential methylation alterations in the tumor groups.

\section{Competing interests}

The authors declare that they have no competing interests.

\section{Authors' contributions}

Experimental design: Y Muto, TM, KS, FK, MP, TR. Pathology analyses: TM. Molecular analyses (i.e., MSI and methylation analyses, bisulfite sequencing, etc.) Y Muto, TM, KS, MS, TK, FW, HK. MS-AFLP Array design: KS, SA. MS-AFLP implementation, data acquisition and analysis: TM, KS, HK, MS, KK, Y Miyaki, SA, MP. Statistical analysis: Y Muto, KS, SA. Experimental work supervision and coordination: KS, FK, MP, TR. Interpretation of the data: Y Muto, TM, KS, TK, SA, MP, TR. Manuscript writing: Y Muto, TM, KS, TK, SA, MP. All authors read and approved the final manuscript.

\section{Acknowledgements}

This work was supported in part by a grant-in-aid for post graduate students from Jichi Medical University, a grant-in-aid from the Ministry of Education, Culture, Sports, Science and Technology, the JKA Foundation through its promotion funds from keirin Racing, and Saitamaken Geka Ikai. MP and SA were supported by grants from the Spanish Institute of Health Carlos III (FIS PI09/2444 and FIS PI12/00511).

\section{Author details}

${ }^{1}$ Department of Surgery, Saitama Medical Center, Jichi Medical University, 1-847, Amanuma-cho, Omiya-ku, Saitama 330-8503, Japan. ${ }^{2}$ First Department of Surgery, Hamamatsu University School of Medicine, 1-20-1, Handa-yama, Higashi-ku, Hamamatsu, Shizuoka 431-3192, Japan. ${ }^{3}$ Nerima-Hikarigaoka Hospital, 2-11-1, Hikarigaoka, Nerima-ku, Tokyo 179-0072, Japan.

${ }^{4}$ Sanford-Burnham Medical Research Institute (SBMRI), 10901 North Torrey Pines Road, La Jolla, California, USA. ${ }^{5}$ Institute of Predictive and Personalized Medicine of Cancer (IMPPC), Carretera de Can Ruti S/N, 08916 Badalona, Barcelona, Spain. ${ }^{6}$ Instituciò Catalana de Recerca i Estudis Avançats (ICREA), Passeig Lluis Companys 23, Barcelona, Spain.

Received: 21 January 2014 Accepted: 16 June 2014

Published: 25 June 2014

\section{References}

1. Fearon ER, Vogelstein B: A genetic model for colorectal tumorigenesis. Cell 1990, 61:759-767.

2. Snover DC, Jass JR, Fenoglio-Preiser C, Batts KP: Serrated polyps of the large intestine: a morphologic and molecular review of an evolving concept. Am J Clin Pathol 2005, 124:380-391.

3. Torlakovic E, Snover DC: Serrated adenomatous polyposis in humans. Gastroenterology 1996, 110:748-755.

4. Longacre TA, Fenoglio-Preiser CM: Mixed hyperplastic adenomatous polyps/serrated adenomas. A distinct form of colorectal neoplasia. Am J Surg Pathol 1990, 14:524-537.

5. Torlakovic E, Skovlund E, Snover DC, Torlakovic G, Nesland JM: Morphologic reappraisal of serrated colorectal polyps. Am J Surg Pathol 2003, 27:65-81.
6. Torlakovic EE, Gomez JD, Driman DK, Parfitt JR, Wang C, Benerjee T, Snover DC: Sessile Serrated Adenoma (SSA) vs. Traditional Serrated Adenoma (TSA). Am J Surg Pathol 2008, 32:21-29.

7. Farris AB, Misdraji J, Srivastava A, Muzikansky A, Deshpande V, Lauwers GY, Mino-Kenudson M: Sessile Serrated Adenoma: Challenging Discrimination From Other Serrated Colonic Polyps. Am J Surg Pathol 2008, 32:30-35.

8. Sandmeier D, Seelentag W, Bouzourene H: Serrated polyps of the colorectum: is sessile serrated adenoma distinguishable from hyperplastic polyp in a daily practice? Virchows Arch 2007, 450:613-618.

9. O'Brien MJ, Yang S, Mack C, Xu H, Huang CS, Mulcahy E, Amorosino M, Farraye FA: Comparison of microsatellite instability, CpG island methylation phenotype, BRAF and KRAS status in serrated polyps and traditional adenomas indicates separate pathways to distinct colorectal carcinoma end points. Am J Surg Pathol 2006, 30:1491-1501.

10. Ionov Y, Peinado MA, Malkhosyan S, Shibata D, Perucho M: Ubiquitous somatic mutations in simple repeated sequences reveal a new mechanism for colonic carcinogenesis. Nature 1993, 363:558-561.

11. Perucho M: Microsatellite instability: the mutator that mutates the other mutator. Nat Med 1996, 2:630-631.

12. Carr NJ, Mahajan H, Tan KL, Hawkins NJ, Ward RL: Serrated and non-serrated polyps of the colorectum: their prevalence in an unselected case series and correlation of BRAF mutation analysis with the diagnosis of sessile serrated adenoma. J Clin Pathol 2009, 62:516-518.

13. Kambara T, Simms LA, Whitehall VL, Spring KJ, Wynter CV, Walsh MD, Barker MA Arnold S, McGivern A, Matsubara N, Tanaka N, Higuchi T, Young J, Jass JR, Leggett BA: BRAF mutation is associated with DNA methylation in serrated polyps and cancers of the colorectum. Gut 2004, 53:1137-1144.

14. Lash RH, Genta RM, Schuler CM: Sessile serrated adenomas: prevalence of dysplasia and carcinoma in 2139 patients. J Clin Pathol 2010, 63:681-686.

15. Sandmeier D, Benhattar J, Martin P, Bouzourene H: Serrated polyps of the large intestine: a molecular study comparing sessile serrated adenomas and hyperplastic polyps. Histopathology 2009, 55:206-213.

16. Yang S, Farraye FA, Mack C, Posnik O, O'Brien MJ: BRAF and KRAS Mutations in hyperplastic polyps and serrated adenomas of the colorectum: relationship to histology and CpG island methylation status. Am J Surg Pathol 2004, 28:1452-1459.

17. Maeda T, Suzuki K, Togashi K, Nokubi M, Saito M, Tsujinaka S, Kamiyama H, Konishi F: Sessile serrated adenoma shares similar genetic and epigenetic features with microsatellite unstable colon cancer in a location-dependent manner. Exp Ther Med 2011, 60:695-700.

18. Issa JP, Shen L, Toyota M: CIMP, at last. Gastroenterology 2005, 129:1121-1124.

19. Toyota M, Ahuja N, Ohe-Toyota M, Herman JG, Baylin SB, Issa JP: CpG island methylator phenotype in colorectal cancer. Proc Natl Acad Sci U S A 1999, 96:8681-8686.

20. Suzuki K, Ohnami S, Tanabe C, Sasaki H, Yasuda J, Katai H, Yoshimura K, Terada M, Perucho M, Yoshida T: The genomic damage estimated by arbitrarily primed PCR DNA fingerprinting is useful for the prognosis of gastric cancer. Gastroenterology 2003, 125:1330-1340.

21. Yamashita K, Dai T, Dai Y, Yamamoto F, Perucho M: Genetics supersedes epigenetics in colon cancer phenotype. Cancer Cell 2003, 4:121-131.

22. Shen $H$, Laird PW: Interplay between the cancer genome and epigenome. Cell 2013, 153:38-55.

23. Suzuki K, Suzuki I, Leodolter A, Alonso S, Horiuchi S, Yamashita K, Perucho M: Global DNA demethylation in gastrointestinal cancer is age dependent and precedes genomic damage. Cancer Cell 2006, 9:199-207.

24. Dong SM, Lee EJ, Jeon ES, Park CK, Kim KM: Progressive methylation during the serrated neoplasia pathway of the colorectum. Mod Pathol 2005, 18:170-178.

25. Grady WM: CIMP and colon cancer gets more complicated. Gut 2007, 56:1498-1500.

26. Park SJ, Rashid A, Lee JH, Kim SG, Hamilton SR, Wu TT: Frequent CpG island methylation in serrated adenomas of the colorectum. Am J Pathol 2003, 162:815-822.

27. Yamamoto F, Yamamoto M, Soto JL, Kojima E, Wang EN, Perucho M, Sekiya T, Yamanaka H: Notl-Msell methylation-sensitive amplied fragment length polymorhism for DNA methylation analysis of human cancers. Electrophoresis 2001, 22:1946-1956.

28. Samuelsson JK, Alonso S, Yamamoto F, Perucho M: DNA fingerprinting techniques for the analysis of genetic and epigenetic alterations in colorectal cancer. Mutat Res 2010, 693:61-76.

29. Kamiyama H, Suzuki K, Maeda T, Koizumi K, Miyaki Y, Okada S, Kawamura YJ, Samuelsson JK, Alonso S, Konishi F, Perucho M: DNA demethylation in 
normal colon tissue predicts predisposition to multiple cancers. Oncogene 2012, 31:5029-5037.

30. Aust DE, Baretton GB: Serrated polyps of the colon and rectum (hyperplastic polyps, sessile serrated adenomas, traditional serrated adenomas, and mixed polyps)-proposal for diagnostic criteria. Virchows Arch 2010, 457:291-297.

31. Higuchi T, Jass JR: My approach to serrated polyps of the colorectum. J Clin Pathol 2004, 57:682-686.

32. Leggett $B$, Whitehall $V$ : Role of the serrated pathway in colorectal cancer pathogenesis. Gastroenterology 2010, 138:2088-2100

33. Chan TL, Zhao W, Leung SY, Yuen ST: BRAF and KRAS mutations in colorectal hyperplastic polyps and serrated adenomas. Cancer Res 2003, 63:4878-4881

34. Davies H, Bignell GR, Cox C, Stephens P, Edkins S, Clegg S, Teague J, Woffendin H, Garnett MJ, Bottomley W, Davis N, Dicks E, Ewing R, Floyd Y, Gray K, Hall S, Hawes R, Hughes J, Kosmidou V, Menzies A, Mould C, Parker A, Stevens C, Watt S, Hooper S, Wilson R, Jayatilake H, Gusterson BA, Cooper C, Shipley J, et al: Mutations of the BRAF gene in human cancer. Nature 2002, 417:949-954

35. Xiong Z, Laird PW: COBRA: a sensitive and quantitative DNA methylation assay. Nucleic Acids Res 1997, 25:2532-2534.

36. Miyaki Y, Suzuki K, Koizumi K, Kato T, Saito M, Kamiyama H, Maeda T, Shibata K, Shiya N, Konishi F: Identification of a potent epigenetic biomarker for resistance to camptothecin and poor outcome to irinotecan-based chemotherapy in colon cancer. Int J Oncol 2012, 40:217-226.

37. Kusano M, Toyota M, Suzuki H, Akino K, Aoki F, Fujita M, Hosokawa M, Shinomura Y, Imai K, Tokino T: Genetic, epigenetic, and clinicopathologic features of gastric carcinomas with the $\mathrm{CpG}$ island methylator phenotype and an association with Epstein-Barr virus. Cancer 2006, 106:1467-1479.

38. Koizumi K, Alonso S, Miyaki Y, Okada S, Ogura H, Shiiya N, Konishi F, Taya T, Perucho M, Suzuki K: Array-based identification of common DNA methylation alterations in ulcerative colitis. Int J Oncol 2012, 40:983-994.

39. Saeed Al, Sharov V, White J, Li J, Liang W, Bhagabati N, Braisted J, Klapa M, Currier T, Thiagarajan M, Sturn A, Snuffin M, Rezantsev A, Popov D, Ryltsov A, Kostukovich E, Borisovsky I, Liu Z, Vinsavich A, Trush V, Quackenbush J: TM4: a free, open-source system for microarray data management and analysis. Biotechniques 2003, 34:374-378.

40. Dehari R: Infrequent APC mutations in serrated adenoma. Tohoku J Exp Med 2001, 193:181-186.

41. Hiyama T, Yokozaki H, Shimamoto F, Haruma K, Yasui W, Kajiyama G, Tahara E: Frequent p53 gene mutations in serrated adenomas of the colorectum. J Pathol 1998, 186:131-139.

42. Jiao YF, Nakamura S, Sugai T, Yamada N, Habano W: Serrated adenoma of the colorectum undergoes a proliferation versus differentiation process: new conceptual interpretation of morphogenesis. Oncology 2008, 74:127-134.

43. Uchida H, Ando H, Maruyama K, Kobayashi H, Toda H, Ogawa H, Ozawa T, Matsuda Y, Sugimura H, Kanno T, Baba S: Genetic alterations of mixed hyperplastic adenomatous polyps in the colon and rectum. Jpn J Cancer Res 1998, 89:299-306.

44. Yamamoto T, Konishi K, Yamochi T, Makino R, Kaneko K, Shimamura T, Ota H, Mitamura K: No major tumorigenic role for beta-catenin in serrated as opposed to conventional colorectal adenomas. $\mathrm{Br} J$ Cancer 2003, 89:152-157.

45. Fu X, Li L, Peng Y: Wnt signalling pathway in the serrated neoplastic pathway of the colorectum: possible roles and epigenetic regulatory mechanisms. J Clin Pathol 2012, 65:675-679.

46. Albuquerque C, Baltazar C, Filipe B, Penha F, Pereira T, Smits R, Cravo M, Lage P, Fidalgo P, Claro I, Rodrigues P, Veiga I, Ramos JS, Fonseca I, Leitao CN, Fodde R: Colorectal cancers show distinct mutation spectra in members of the canonical WNT signaling pathway according to their anatomical location and type of genetic instability. Genes Chromosomes Cancer 2010, 49:746-759.

47. Zeng L, Fagotto F, Zhang T, Hsu W, Vasicek TJ, Perry WL 3rd, Lee JJ, Tilghman SM Gumbiner BM, Costantini F: The mouse Fused locus encodes Axin, an inhibitor of the Wnt signaling pathway that regulates embryonic axis formation. Cell 1997, 90:181-192.

48. Taniguchi K, Roberts LR, Aderca IN, Dong X, Qian C, Murphy LM, Nagorney DM, Burgart LI, Roche PC, Smith DI, Ross JA, Liu W: Mutational spectrum of beta-catenin, AXIN1, and AXIN2 in hepatocellular carcinomas and hepatoblastomas. Oncogene 2002, 21:4863-4871.
49. Liu W, Dong X, Mai M, Seelan RS, Taniguchi K, Krishnadath KK, Halling KC, Cunningham JM, Boardman LA, Qian C, Christensen E, Schmidt SS, Roche PC, Smith DI, Thibodeau SN: Mutations in AXIN2 cause colorectal cancer with defective mismatch repair by activating beta-catenin/TCF signalling. Nat Genet 2000, 26:146-147.

50. Lustig B, Jerchow B, Sachs M, Weiler S, Pietsch T, Karsten U, van de Wetering M, Clevers H, Schlag PM, Birchmeier W, Behrens J: Negative feedback loop of Wnt signaling through upregulation of conductin/ axin2 in colorectal and liver tumors. Mol Cell Biol 2002, 22:1184-1193.

51. Yan D, Wiesmann M, Rohan M, Chan V, Jefferson AB, Guo L, Sakamoto D, Caothien RH, Fuller JH, Reinhard C, Garcia PD, Randazzo FM, Escobedo J, Fantl WJ, Williams LT: Elevated expression of axin2 and hnkd mRNA provides evidence that Wnt/beta -catenin signaling is activated in human colon tumors. Proc Natl Acad Sci U S A 2001, 98:14973-14978.

52. Koinuma K, Yamashita Y, Liu W, Hatanaka H, Kurashina K, Wada T, Takada S, Kaneda R, Choi YL, Fujiwara SI, Miyakura Y, Nagai H, Mano H: Epigenetic silencing of AXIN2 in colorectal carcinoma with microsatellite instability. Oncogene 2006, 25:139-146.

53. Thorstensen L, Lind GE, Lovig T, Diep CB, Meling Gl, Rognum TO, Lothe RA: Genetic and epigenetic changes of components affecting the WNT pathway in colorectal carcinomas stratified by microsatellite instability. Neoplasia 2005, 7:99-108.

54. Yamamoto H, Imai K, Perucho M: Gastrointestinal cancer of the microsatellite mutator phenotype pathway. J Gastroenterol 2002, 37:153-163.

55. Kane MF, Loda M, Gaida GM, Lipman J, Mishra R, Goldman H, Jessup JM, Kolodner R: Methylation of the hMLH1 promoter correlates with lack of expression of hMLH1 in sporadic colon tumors and mismatch repair-defective human tumor cell lines. Cancer Res 1997, 57:808-811.

56. Toyota M, Ho C, Ahuja N, Jair KW, Li Q, Ohe-Toyota M, Baylin SB, Issa JP. Identification of differentially methylated sequences in colorectal cancer by methylated CpG island amplification. Cancer Res 1999, 59:2307-2312.

57. Kim YH, Kakar S, Cun L, Deng G, Kim YS: Distinct CpG island methylation profiles and BRAF mutation status in serrated and adenomatous colorectal polyps. Int J Cancer 2008, 123:2587-2593.

58. O'Brien MJ, Yang S, Clebanoff JL, Mulcahy E, Farraye FA, Amorosino M, Swan N: Hyperplastic (serrated) polyps of the colorectum: relationship of CpG island methylator phenotype and K-ras mutation to location and histologic subtype. Am J Surg Pathol 2004, 28:423-434.

doi:10.1186/1471-2407-14-466

Cite this article as: Muto et al:: DNA methylation alterations of AXIN2 in serrated adenomas and colon carcinomas with microsatellite instability. BMC Cancer 2014 14:466.

\section{Submit your next manuscript to BioMed Central and take full advantage of:}

- Convenient online submission

- Thorough peer review

- No space constraints or color figure charges

- Immediate publication on acceptance

- Inclusion in PubMed, CAS, Scopus and Google Scholar

- Research which is freely available for redistribution
C) Biomed Central 